\title{
Recommendations on Training in Social and Community Psychiatry in SHO/Registrar training schemes
}

\author{
Section for Social and Community Psychiatry
}

1. These recommendations are derived from the report of a working party on education and training set up by the Section for Social and Community Psychiatry in 1984. A companion paper deals with recommendations for Senior Registrar posts in Rehabilitation and in General Psychiatry. The recommendations for the most part do not represent new departures, but are mainly explicit descriptions of what is currently included in good training programmes, developments which are already increasing or recommendations made in previous documents. With the forthcoming expansion of psychiatric care in the community, appropriate training is increasingly required.

2. Social and Community Psychiatry comprises an important aspect of the work of all general psychiatrists, and is central to much of the organisation and practice of comprehensive care to a catchment area. The subject matter is broad, incorporating social aspects of aetiology and presentation of disorder, of treatment, and the social consequences of disorder. DHSS policy is that psychiatrists should be working more in the community. A recent survey revealed that already $20 \%$ of psychiatric teams do some work in primary care and thus are involved increasingly with minor in addition to major disorders.

3. Didactic courses and seminar programmes should include a critical presentation of some of the following topics: social factors in causation including life events, social support, social correlates of disorder; epidemiology; understanding of relevant aspects of social environment; transcultural psychiatry; social aspects of treatment; rehabilitation; community care; crisis intervention; use of community facilities such as hostels, day centres, sheltered workshops; therapeutic communities; organisation and future evolution of services; relationships with other disciplines and services such as social services, community psychiatric nursing, community medicine, self help and voluntary bodies; consultation methods; psychiatry in primary care; administration and management; management of change; consumer satisfaction; prevention; evaluation and research methods.

4. In training experiences, every general professional SHO/Registrar rotational training programme should have within it opportunities for experience in the following. although not each should be mandatory for every trainee. It is desirable for all trainees to have experience of (a)-(c), preferably during SHO/Registrar training. Deficiencies may be remedied at Senior Registrar level.

(a) a structured progressive rehabilitation programme spanning a range of settings including not only inpatient wards, but day facilities such as day centres and sheltered workshops and liaison with hostels, supervised lodgings;

(b) care of the long term mentally disordered;

(c) close liaison with a team which includes community psychiatric nurses;

(d) psychiatric work in a primary care setting;

(e) where possible a team which has a domiciliary approach not only to assessment but to treatment.

\section{Approval of posts}

All other Colleges inspect individual posts. Ours inspects schemes. This is because we have a special concern for the provision of a balanced and varied training programme. But a scheme is as good as the sum of its parts. What does the Approval Panel do when it encounters a training post which falls below the College's standard? First, the Panel does its best to discount local rivalries and the occasional dissatisfied trainee. The evidence has to be good.

Secondly, it has to make some sort of comment on this state of affairs, often informally, but sometimes in its report. Previously, such comments were of a judiciously general nature-often not much use to the local tutors. Or else a whole scheme was penalised because of a poor post - rather a damaging solution. In the past few years the College's comments have become rather more specific and some individual posts have lost their approval. This has inevitably led to some hurt feelings, but it is, I believe, the best and most honest attempt to deal with this issue.

The General Medical Council's recent suggestions for 'Designated Trainers', and the general contraction of Registrar posts, both point in the same direction: in future, training posts, as well as schemes, will be subject to very careful scrutiny.

DR J. L. T. BIRLEY Dean February 1987 